\title{
Expression and functional analysis of Nr2e3, a photoreceptor-specific nuclear receptor, suggest common mechanisms in retinal development between avians and mammals
}

\author{
Mime Kobayashi $\cdot$ Kenji Hara $\cdot$ Ruth T. Yu • \\ Kunio Yasuda \\ Received: 7 March 2008 / Accepted: 30 May 2008 / Published online: 1 July 2008 \\ (C) The Author(s) 2008
}

\begin{abstract}
The photoreceptor-specific nuclear receptor (PNR; Nr2e3) is a transcription factor important for retinal development. We report here the identification and expression analysis of the avian $\mathrm{Nr} 2 \mathrm{e} 3 . N r 2 e 3$ mRNA is expressed in the photoreceptor layer of the neural retina during early stages of chick embryogenesis. Its temporal expression is distinct from that of a related nuclear receptor, Tlx. Chick Nr2e3 recognizes and binds to the same target DNA sequence as its vertebrate orthologs. Functional assays revealed that chick $\mathrm{Nr} 2 \mathrm{e} 3$ acts as a transcriptional repressor. Our results suggest that $\mathrm{Nr} 2 \mathrm{e} 3$ plays a common role in retinal development in vertebrates.
\end{abstract}

Communicated by C. Desplan

The sequence reported in this paper has been deposited in the GenBank database [accession no. AY053465 (chicken Nr2e3)].

M. Kobayashi $(\bowtie) \cdot$ K. Yasuda

Graduate School of Biological Sciences,

Nara Institute of Science and Technology,

8916-5 Takayama,

Ikoma, Nara 630-0192, Japan

e-mail: kobayashi.mime01@bs.naist.jp

M. Kobayashi $\cdot$ K. Hara $\cdot$ R. T. Yu

Institute for Virus Research, Kyoto University,

53 Shogoin Kawahara-cho, Sakyo-ku,

Kyoto 606-8507, Japan

Present address:

K. Hara

Tokyo Gakugei University,

Tokyo, Japan

Present address:

R. T. Yu

Gene Expression Laboratory,

The Salk Insitute for Biological Studies,

La Jolla, CA, USA
Keywords Nuclear receptor - Transcription factor . Eye development . Chicken

\section{Introduction}

Transcription factor proteins regulate gene expression by binding to specific DNA sequences in regulatory regions of the gene. Nuclear receptors have a unique modular structure, with a characteristic ligand-binding domain in addition to their DNA-binding domain (Mangelsdorf et al. 1995). They are receptors for lipophilic molecules such as steroid and thyroid hormones, vitamins A and D, and at the same time, they function as transcription factors. Many nuclear receptors are known to regulate cell fate determination. However, ligands have not been identified for a substantial number of nuclear receptors and members of this class are called orphan receptors (Benoit et al. 2006).

Photoreceptor-specific nuclear receptor (PNR; Nr2e3) is one of these orphan receptors, whose expression is restricted almost exclusively to the photoreceptor layer of the neural retina (Kobayashi et al. 1999; Chen et al. 2005; Kitambi and Hauptmann 2007; Martinez-De Luna and ElHodiri 2007; McIlvain and Knox 2007). Mutations of this gene have been implicated in several forms of retinal disorder (Gerber et al. 2000 and references in Chen et al. 2005). Detailed analyses of its function have been conducted using various model vertebrate systems and suggested that $\mathrm{Nr} 2 \mathrm{e} 3$ is important in the development and maintenance of rod photoreceptor cells (Chen et al. 2005; Mcllvain and Knox 2007; Oh et al. 2008).

Chick embryos have long been a favorite model for performing functional analyses of transcription factor proteins. It allows observation of retinal development in 
vivo with relative ease and the availability of gene transfer methods such as electrophoresis is an additional advantage (Stern 2005). Identification of the chick Nr2e3 gene is, thus, a useful tool to further these studies.

We used a cDNA library from embryonic day 8 (E8) chick retina to identify an avian $N r 2 e 3$ clone. The mRNA expression of this chick $\mathrm{Nr} 2 \mathrm{e} 3$ was found to be restricted in the neural retina. We analyzed its expression during a developmental time course along with a related transcription factor, Tlx. Structurally, the chick Nr2e3 shares an asparagine substitution in the P box of its DNA-binding domain with its vertebrate orthologs in contrast to the acidic amino acid residue present in other nuclear receptor family members. Accordingly, the Nr2e3 proteins show the same DNAbinding preferences, suggesting that they regulate a similar set of target genes. Transcription assays further confirmed that chick $\mathrm{Nr} 2 \mathrm{e} 3$ function as a transcriptional repressor.

\section{Materials and methods}

\section{RNA extraction}

Fertilized chicken eggs were obtained from Okada Farm (Nara, Japan) and incubated at $35^{\circ} \mathrm{C}$ for up to 14 days. The embryos were harvested, tissues were dissected, and total RNA was extracted using ISOGEN (Nippon Gene, Toyama, Japan) following the manufacturer's protocol.

\section{Identification of the chicken $\mathrm{Nr} 2 \mathrm{e} 3$ ortholog}

Using total RNA extracted from the neural retina of E8 chick embryos, mRNA was purified with Oligotex ${ }^{\mathrm{TM}_{-} \mathrm{dT} 30}$ $<$ Super $>$ Kit (Roche). A lambda phage cDNA library was constructed using the Lambda System for cDNA Cloning (Life Technologies) and Gigapack III Gold Packaging extract (Stratagene). A polymerase chain reaction fragment corresponding to full-length human $\mathrm{Nr} 2 \mathrm{e} 3$ (amino acids 8 to poly(A)+ region) was amplified from a human PNR (Nr2e3) plasmid construct using primers NMO70 (5'TCTGATGAGCTCCACAGTGGCTGC-3') and T3 (5'GCAATTAACCCTCACTAAAGGG-3') (Kobayashi et al. 1999). The DNA fragment was gel-purified, labeled with alpha- ${ }^{32} \mathrm{P}-\mathrm{dCTP}$ using Megaprime DNA Labeling System (Amersham Pharmacia) and used as a probe to screen $0.8 \times$ $10^{5}$ independent clones in the E8 retinal library using an ExAssist helper phage (Stratagene). Twenty-seven positive clones were identified and further analyzed in a second screening using the same probe. Fifteen strongly positive clones were selected for DNA sequencing and three potential chicken $N r 2 e 3$ clones were identified. The most $5^{\prime}$ end, which corresponded to the region between the DNA- and ligand-binding domain of the potential chicken
Nr2e 3 was amplified using primers KMO40 (5'-TAGA CAGCATTGAGCTGGACGCCGA-3') and KMO22 (5'CGTGCTGAGGTCTCGTAGACGTT-3') and another screening was performed. Independent clones of $2.0 \times 10^{5}$ were screened and nine positive clones were identified. After a second screening using the same probe, three positive clones were identified and DNA sequence was determined using an ALF express sequencing machine (Amersham Pharmacia).

Northern blotting and in situ hybridization

A $0.75-\mathrm{kb}$ cDNA fragment of chicken $N r 2 e 3$ [nucleotides 177-915] was amplified using primers KMO6 (5'CCAGTGGACAAGGCACACCGCAAC-3') and KMO22 and labeled with digoxigenin (Boehringer; for $\mathrm{Nr} 2 e 3$ probe), or amplified using KMOcT5 (5'-ATCCAGGCTT TACAGGAGGTTGTGG-3') and KMOcT3 (5'-TCCG AAACGACAGGGTTGTGTAGG-3') and labeled with alpha- ${ }^{32} \mathrm{P}$-dCTP (for $T l x$ probe). Gel electrophoresis, membrane transfer, and hybridization conditions were as described (Kobayashi et al. 1999). A nylon membrane blot containing $10 \mu \mathrm{g}$ of each total RNA was prepared. The $18 \mathrm{~S}$ and $28 \mathrm{~S}$ rRNA on the membrane was visualized by $5 \%$ methylene blue in 10\% acetic acid and used as loading control. After hybridization with the Nr2e3 probe, the signals were detected using an ECL Detection System (Amersham Pharmacia). Subsequently, the blot was rehybridized with a $T l x$ probe and the signal was visualized on $\mathrm{X}$-ray film. In situ hybridization of frozen tissue sections was performed as described (Kobayashi et al. 1999) using a 0.75-kb digoxygenin-labeled antisense RNA probe.

\section{DNA-binding assays}

The chick $\mathrm{Nr} 2 \mathrm{e} 3$ protein was translated from a pCMX cNr2e3 expression vector (Kobayashi et al. 1999) using T7 RNA polymerase and a TNT ${ }^{\circledR}$ coupled rabbit reticulocyte in vitro translation kit (Promega). Probe-labeling and gel shift assay was performed as described (Kobayashi et al. 1999). The sequences of double-stranded probes used for the assays are indicated in the figure with $5^{\prime}$-agct hangovers.

Transactivation assays

Expression plasmids for the GAL4-Nr2e3 fusion protein were obtained by ligating cDNA fragments encoding chick (Arg 92 to stop codon) or human (Met 113 to stop codon) $\mathrm{Nr} 2 \mathrm{e} 3$ ligand-binding domain into a cytomegalovirus promoter-driven plasmid pCMX-GAL4 (Gerber et al. 2000). CV-1 cells were used for transfection assays in 12well tissue culture plates by calcium phosphate precipitation (Umesono and Evans 1989). Cells were maintained in 
Dulbecco's modified Eagle's medium (DMEM) supplemented with $10 \%$ bovine calf serum (BCS). Transfection mixtures contained $100 \mathrm{ng}$ of receptor expression plasmid, $250 \mathrm{ng}$ of MH100 $\times 4$-tk-luc reporter plasmid and $500 \mathrm{ng}$ of pCMX- $\beta$ GAL as control for transfection efficiency. Cells were exposed to the precipitate for 6-8 h, washed, and treated with phenol red-free DMEM, 10\% charcoal-resintreated BCS, before harvesting and assaying for luciferase and $\beta$-galactosidase activity. All points were performed in triplicate and repeated at least twice in independent experiments with variations of less than $10 \%$.

\section{Results and discussion}

Identification of the chicken $\mathrm{Nr} 2 e 3$ gene

In vertebrates including humans and mice, the $N r 2 e 3$ gene is known to be exclusively expressed in the neural retina (Kobayashi et al. 1999; Chen et al. 2005; Kitambi and
Hauptmann 2007; Martinez-De Luna and El-Hodiri 2007; McIlvain and Knox 2007). Therefore, to identify a chick Nr2e3 cDNA, we constructed a phage cDNA library from E8 chick neural retina. A total of $0.3 \times 10^{6}$ independent clones were screened and six clones that showed a high homology to mammalian $N r 2 e 3$ were identified. The deduced amino acid sequence of chick $\mathrm{Nr} 2 \mathrm{e} 3$ showed $70 \%$ homology to its mammalian counterparts, with even more similarity to the amphibian Nr2e3 (Fig. 1a; Kobayashi et al. 1999; Chen et al. 2005; Martinez-De Luna and ElHodiri 2007). Three other clones, which showed relatively high hybridization signals to the $N r 2 e 3$ probe, were found to encode $N r 2 e l(T l x)$ and $N r 2 f(C O U P-T F)$ family genes, which are most closely related to $N r 2 e 3$ among the nuclear receptor superfamily (Benoit et al. 2006). Translation of the Nr2e3 protein is assumed to initiate from the first ATG codon found in the cloned mRNA because an in-frame stop codon (TAA) was found 80 bp upstream of the initiation codon in Gallus gallus whole genome shotgun sequence pqd72h09.b1 (accession number 140842551; Fig. 1b). In
Fig. 1 a Sequence alignment of chicken, Xenopus, zebrafish, and human Nr2e3. DNA- and ligand-binding domains are shaded. The position of the first intron and the $\mathrm{P}$ box are indicated by an arrowhead and a box. The dashed underline indicates the region used as a probe for northern and in situ hybridization analyses.

b Primary structure of the $5^{\prime}$ region of the chicken $\mathrm{Nr} 2 \mathrm{e} 3$ gene. The sequence found in cDNA clone(s) is underlined with the $5^{\prime}$ end indicated by asterisk. The first ATG is in bold, with an in-frame upstream stop codon (bold-underlined). The splice donor sequence (GT) is shown in italics a $\mathrm{CNR} 2 \mathrm{E} 3$ XNR2E3 zNR2E3 hNR2E3

CNR2E3 XNR2E3 zNR2E3 hNR2E3

CNR2E3 xNR2E3 zNR2E3 hNR2E3

CNR2E3 XNR2E3 zNR2E3 hNR2E3

CNR2E3 XNR2E3 zNR2E3 hNR2E3

CNR2E3 XNR2E3 zNR2E3 hNR2E3

CNR2E3 XNR2E3 ZNR2E3 hNR2E3

CNR2E3 XNR2E3 zNR2E3 hNR2E3

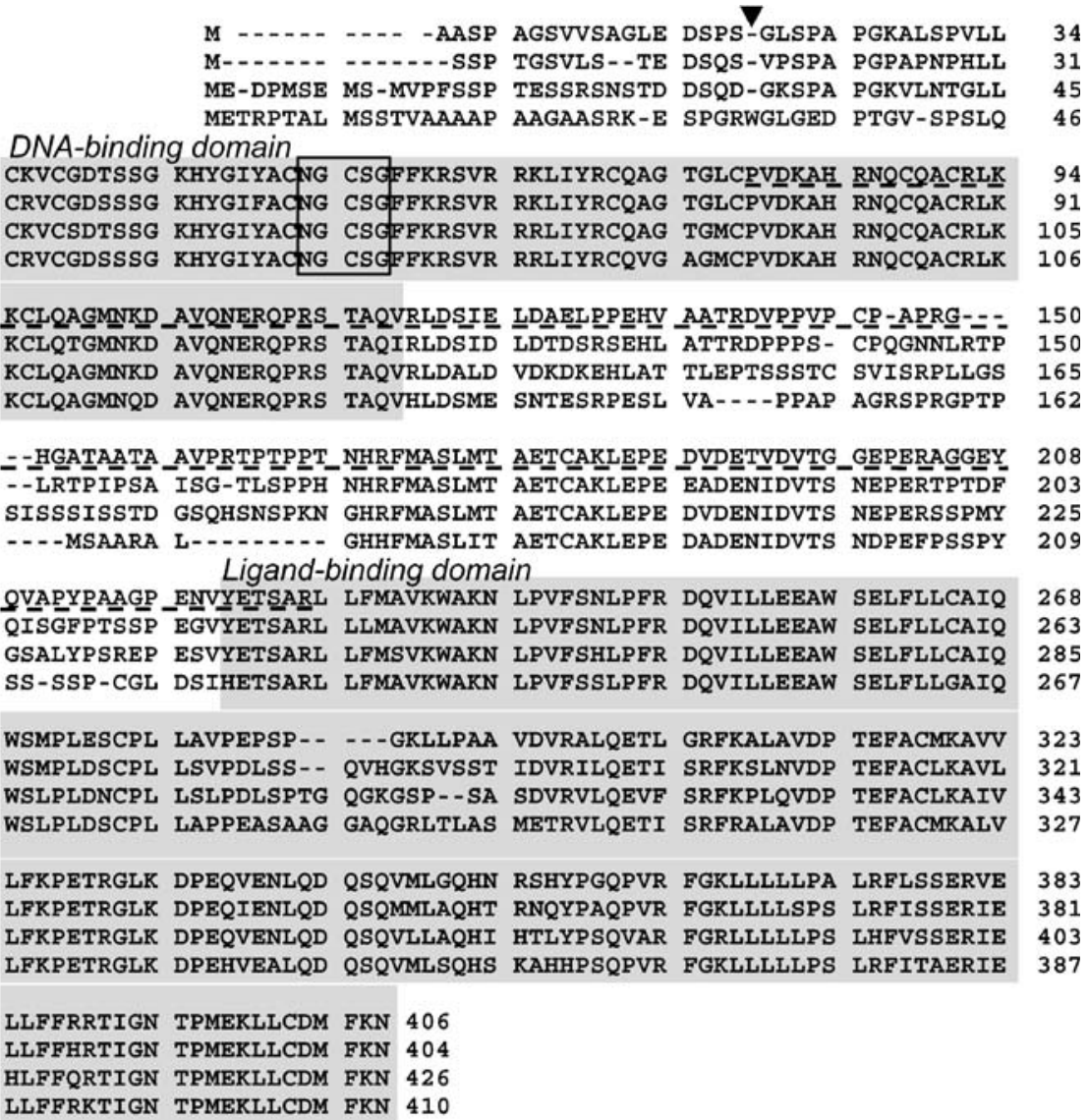

M - - AASP AGSVVSAGLE DSPS-GLSPA PGKALSPVLL 34 M-ME-DPMSE MS-MVPFSSP TESSRSNSTD DSQD-GKSPA PGKVLNTGLL 45 METRPTAL MSSTVAAAAP AAGAASRK-E SPGRWGLGED PTGV-SPSLQ DNA-binding domain CRVCGDSSSG KHYGIYACNG CSGPFKRSVR RRLIYRCQVG AGMCPVDKAH RNQCQACRLK

KCLLAGMNKD_AVQNEROPRS_TAQVRLDSIE LDAELPPPEHV _AATRDVPPVP_CP-APRG-:KCLA 08 03 68 63 185 7 1 3 81 03

-80 CACAGCGCCC GCGCTAATCT GCCTTGAAGA GAGCACAGCC ACAGCCCCAT CCTGCAGAGC - 1

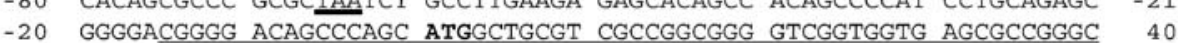

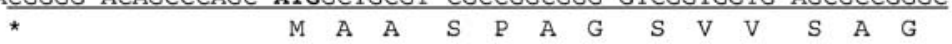

41 TAGAGGACAG CCCCTCGGGT AAGAGCCACA TGCTGCTGCA CAGGTCTGTC CCCAGCGGT 100

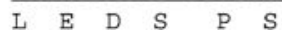




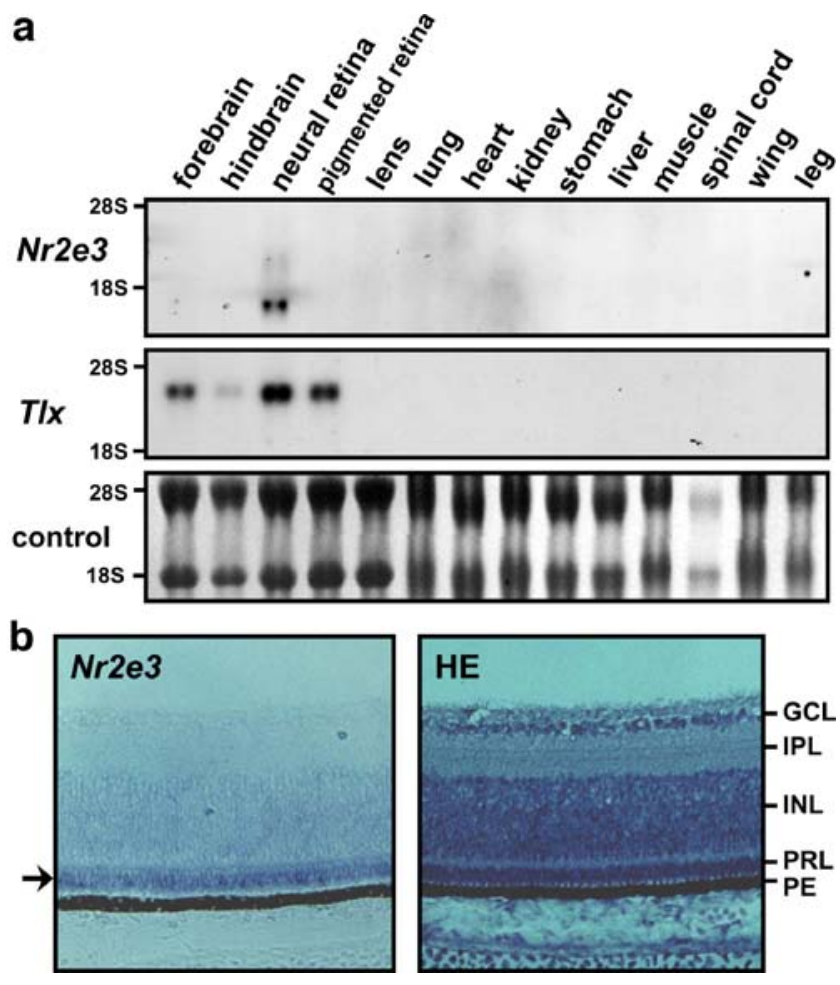

Fig. 2 Expression of $N r 2 e 3$ mRNA in chicken. a Northern hybridization analyses of mRNA from E8 chick embryo tissues/organs using the chicken $N r 2 e 3$ or Tlx cDNA as probes. All lanes contain $10 \mu \mathrm{g}$ of total RNA. For $\mathrm{Nr} 2 e 3$, strong signal was detected around $1.7 \mathrm{~kb}$ and relatively weak signal of $2.6 \mathrm{~kb}$. The $18 \mathrm{~S}$ and $28 \mathrm{~S}$ rRNA were used as a loading control. b In situ hybridization of E14 chick embryo retina sections with digoxigenin-labeled $N r 2 e 3$ RNA probe. The arrow indicates $N r 2 e 3$ signal in the photoreceptor layer. An adjacent section was stained with hematoxylin-eosin $(H E)$ to help visualize different cell layers. $G C L$ Ganglion cell layer, $I P L$ inner plexiform layer, $I N L$ inner nuclear layer, $P R L$ photoreceptor layer, $P E$ pigment epithelium

addition, a putative splice donor sequence (gt) for the first intron is found at the same position where the first intron is located in the zebrafish $\mathrm{Nr} 2 e 3$ gene (Chen et al. 2005).

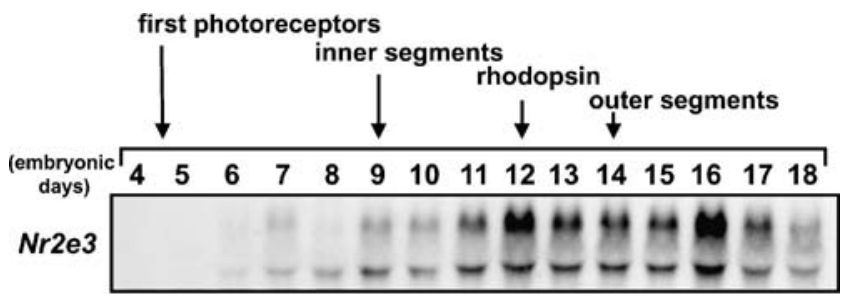

$T / x$

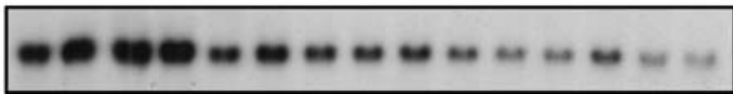

Fig. 3 Temporal expression of $N r 2 e 3$ and Tlx mRNA in early retinal development. Northern hybridization analyses of mRNA from the neural retina of chick embryos at different stages of development using the chick $N r 2 e 3$ or $T l x$ cDNA as a probe. All lanes contain $10 \mu \mathrm{g}$ of total RNA. Approximate timing of events in eye development are indicated

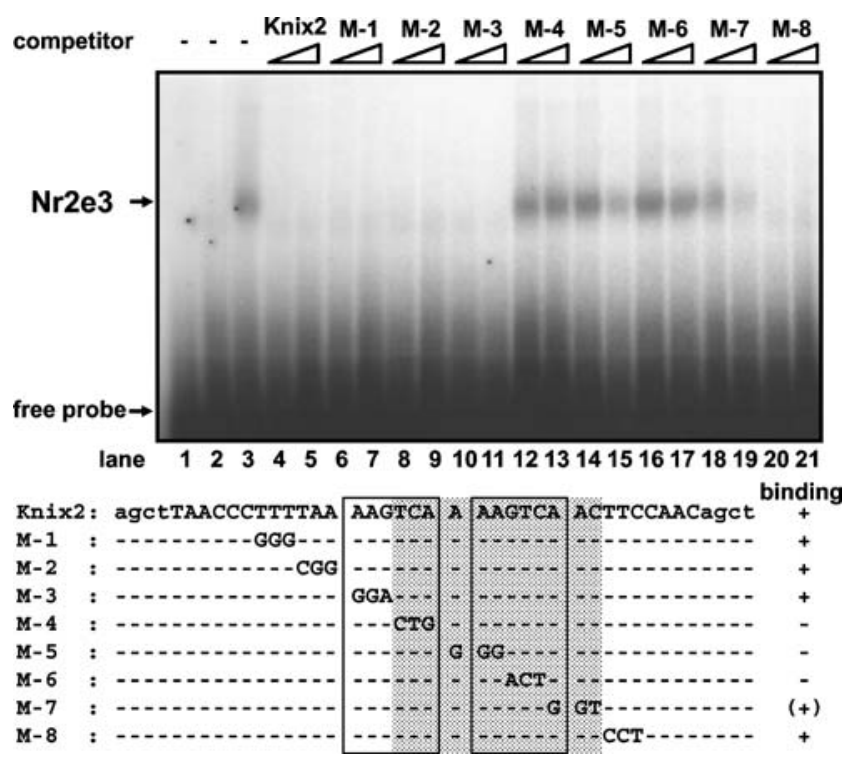

Fig. 4 In vitro DNA binding preference by Nr2e3 protein. A gel mobility shift assay was performed. Lane 1 is probe only, and mock cell extract was added in lane 2. Lane 3 shows a specific proteinDNA complex formed when $\mathrm{Nr} 2 \mathrm{e} 3$ protein is added. Lanes $4-11$ and 19-21 show that $\mathrm{Nr} 2 \mathrm{e} 3$ bound to labeled Kni $\mathrm{x} 2$ probe can be blocked by competition by addition of excess mutant cold probes shown below. Competitor probes are indicated above, with ten-fold excess added in lanes 4, 6, 8, 10,12,14,16,18,20, and 50-fold excess in lanes $5,7,9,11,13,15,17,19,21$. Shaded region indicates the nucleotide sequence shown to be important for recognition by $\mathrm{Nr} 2 \mathrm{e} 3$ protein. The tandem repeat of AAGTCA motifs in Kni x2 probe are boxed

Nr2e3 mRNA is expressed in photoreceptor cells of neural retina

Total RNA was extracted from different tissues of E8 chick embryos and the pattern of Nr2e3 mRNA expression was analyzed. Nr2e3 expression was observed only in the neural retina among the organs examined, while mRNA of the related Tlx (Yu et al. 2000) was also expressed in the brain and pigmented retina (Fig. 2a). Using RNA from E14 chick embryos, the restricted expression of $\mathrm{Nr} 2 e 3$ in the neural retina was confirmed (data not shown). In situ hybridization of E14 chick retina sections revealed that $\mathrm{Nr} 2 e 3$ expression is restricted to the photoreceptor layer of neural retina (Fig. 2b). These results confirm that the clone we identified

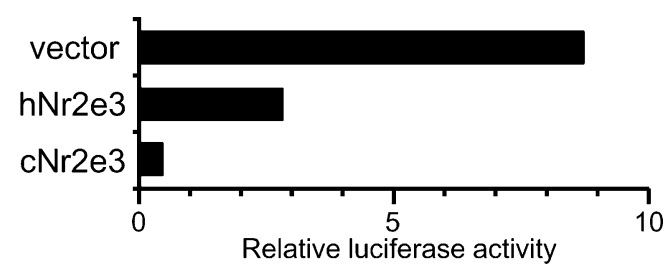

Fig. $5 \mathrm{Nr} 2 \mathrm{e} 3$ protein represses transcription. Transient transfection assays in $\mathrm{CV}-1$ cells show that the chick $\mathrm{Nr} 2 \mathrm{e} 3$ can repress the activity of a luciferase reporter gene to a greater extent than human $\mathrm{Nr} 2 \mathrm{e} 3$ 
in chicken is indeed an ortholog of the mammalian $\mathrm{Nr} 2 e 3$. To elucidate the potential role of $N r 2 e 3$ in retinal development, its mRNA expression was examined using RNA extracted from neural retina at different stages (Fig. 3). The expression of $N r 2 e 3$ increased in the later stages of embryogenesis, while the expression of $T l x$ was the highest in the early stages (E5 to E7) and gradually declined (Fig. 3). Considering that $N r 2 e 3$ is almost exclusively expressed in the photoreceptor layer, it is interesting that the expression of $N r 2 e 3$ mRNA gradually increases as photoreceptor cells develop.

\section{DNA-binding specificity of $\mathrm{Nr} 2 \mathrm{e} 3$}

The first amino acid in the Nr2e3 P box of its DNA-binding domain (Umesono and Evans 1989), which is critical for recognition of target DNA sequence, is an asparagine $(\mathrm{N})$ as is the case for other Nr2e 3 orthologs (Fig. 1a; Kobayashi et al. 1999; Chen et al. 2005). This is a unique feature among nuclear receptors and it has been shown that mammalian and zebrafish $\mathrm{Nr} 2 \mathrm{e} 3$ bind to a tandem repeat of AAGTCA motifs separated by one nucleotide (Kobayashi et al. 1999; Chen et al. 2005). To examine the binding specificity of chick $\mathrm{Nr} 2 \mathrm{e} 3$ protein, a gel mobility shift assay was performed using a probe to which mammalian Nr2e3 protein was shown to bind. Chick Nr2e3 showed the same binding preferences with its mammalian counterpart (Fig. 4). Competition experiments further revealed that nucleotides important for recognition by mammalian $\mathrm{Nr} 2 \mathrm{e} 3$ were also conserved in chick $\mathrm{Nr} 2 \mathrm{e} 3$ and necessary for its target DNA specificity (Fig. 4).

\section{Function of Nr2e3}

In earlier studies, mammalian $\mathrm{Nr} 2 \mathrm{e} 3$ was shown to repress transcription of its target genes (Gerber et al. 2000; Chen et al. 2005). To test the transcriptional activity of the chick $\mathrm{Nr} 2 \mathrm{e} 3$, a plasmid coding for the GAL4 DNA-binding domain fused to the Nr2e3 ligand-binding domain was constructed and cotransfected with a reporter plasmid controlled by a promoter containing GAL4-binding sites (Fig. 5). Along with human Nr2e3, we confirmed that the chick Nr2e3 acts as a transcriptional repressor (Fig. 5).

The role of the $N r 2 e 3$ gene in vertebrates has been widely analyzed (Gerber et al. 2000; Chen et al. 2005; McIlvain and Knox 2007; Oh et al. 2008) and suggests a common function for $N r 2 e 3$ in those species. Recently, a $N r 2 e 3$ ortholog was reported in the genome of the gray, short-tailed opossum (Monodelphis domestica) published as the first metatherian ('marsupial') species to be sequenced (GenBank accession number XM_001371384). In addition, putative Nr2e3 orthologs have been found in Caenorhabditis elegans (Kobayashi et al. 1999; Wightman et al. 2005) and sea urchin Strongylocentrotus purpuratus (GenBank accession number XP_780706). The C. elegans counterpart, FAX-1, also contains an asparagine in its $\mathrm{P}$ box and has shown to be important for axon pathfinding and specification of neuron identities (Wightman et al. 2005). Considering the restricted expression of $\mathrm{Nr} 2 \mathrm{e} 3$ in the vertebrate neural retina, it is interesting to investigate the function of $\mathrm{Nr} 2 \mathrm{e} 3$ in those species that do not have apparent organ comparable to a vertebrate eye. Potential molecules that may modulate Nr2e3 function have also been reported, adding a tool to investigate the function of Nr2e3 (Wolkenberg et al. 2006).

Regarding factors that might control Nr2e3 expression, a Maf protein $\mathrm{Nrl}$ has been suggested for its regulation (Oh et al. 2008). Although $\mathrm{Nrl}$ is not found in chicken, another Maf protein, L-Maf has been found to be expressed in rod cells in the later stages (Ochi et al. 2004). It is possible that L-Maf or other Maf protein(s) may regulate Nr2e3 expression in chicken.

In conclusion, we succeeded in identifying a chicken ortholog of the $N r 2 e 3$ gene, whose mRNA expression is found only in the photoreceptor layer of the neural retina. During chick development, Nr2e3 expression was high in the later stages (E12 to E17) while the expression of closely related Tlx gene was high in the early stages (E5 to E7). The binding specificity of the avian $\mathrm{Nr} 2 \mathrm{e} 3$ protein shares the same preferences as its vertebrate orthologs and also functions as a transcriptional repressor. Our results suggest that $\mathrm{Nr} 2 \mathrm{e} 3$ has a common function in retinal development that is shared between avians and mammals.

Acknowledgements The authors would like to acknowledge that this project was initiated and mainly conducted under the direct supervision of late Professor Kazuhiko Umesono at Kyoto University. The authors thank Dr. Yoshihiko Umesono for sharing unpublished results and Dr. Shin-ichiro Takezawa for plasmid construction. This work was supported in part by grants from the Human Frontier Science Program; the Research for the Future Program of the Japan Society for the Promotion of Science; Grants-in-Aid for Scientific Research from the Ministry of Education, Science, Sports, and Culture of Japan; and the Foundation for Nara Institute of Science and Technology.

Open Access This article is distributed under the terms of the Creative Commons Attribution Noncommercial License which permits any noncommercial use, distribution, and reproduction in any medium, provided the original author(s) and source are credited.

\section{References}

Benoit G, Cooney A, Giguere V, Ingraham H, Lazar M, Muscat G, Perlmann T, Renaud JP, Schwabe J, Sladek F, Tsai MJ, Laudet V (2006) International Union of Pharmacology. LXVI. Orphan nuclear receptors. Pharmacol Rev 58:798-836

Chen J, Rattner A, Nathans J (2005) The rod photoreceptor-specific nuclear receptor $\mathrm{Nr} 2 \mathrm{e} 3$ represses transcription of multiple conespecific genes. J Neurosci 25:118-129 
Gerber S, Rozet JM, Takezawa S-I, dos Santos LC, Lopes L, Gribouval O, Penet C, Perrault I, Ducroq D, Souied E, Jeanpierre M, Romana S, Frezal J, Ferraz F, Yu-Umesono R, Munnich A, Kaplan J (2000) The photoreceptor cell-specific nuclear receptor gene (PNR) accounts for retinitis pigmentosa in the Crypto-Jews from Portugal (Marranos), survivors from the Spanish inquisition. Hum Genet 107:276-284

Kitambi SS, Hauptmann G (2007) The zebrafish orphan nuclear receptor genes $\mathrm{Nr} 2 \mathrm{e} 1$ and $\mathrm{Nr} 2 \mathrm{e} 3$ are expressed in developing eye and forebrain. Gene Expr Patterns 7:521-528

Kobayashi M, Takezawa S, Hara K, Yu RT, Umesono Y, Agata K, Taniwaki M, Yasuda K, Umesono K (1999) Identification of a photoreceptor cell-specific nuclear receptor. Proc Natl Acad Sci USA 96:4814-4819

Mangelsdorf DJ, Thummel C, Beato M, Herrlich P, Schutz G, Umesono K, Blumberg B, Kastner P, Mark M, Chambon P, Evans RM (1995) The nuclear receptor superfamily - the second decade. Cell 83:835-839

Martinez-De Luna RI, El-Hodiri HM (2007) The Xenopus ortholog of the nuclear hormone receptor $\mathrm{Nr} 2 \mathrm{e} 3$ is primarily expressed in developing photoreceptors. Int J Dev Biol 51:235-239

McIlvain VA, Knox BE (2007) Nr2e3 and Nrl can reprogram retinal precursors to the rod fate in Xenopus retina. Dev Dyn 236:1970 1979
Ochi H, Sakagami K, Ishii A, Morita N, Nishiuchi M, Ogino H, Yasuda K (2004) Temporal expression of L-Maf and RaxL in developing chicken retina are arranged into mosaic pattern. Gene Expr Patterns 4:489-494

Oh EC, Cheng H, Hao H, Jia L, Khan NW, Swaroop A (2008) Rod differentiation factor NRL activates the expression of nuclear receptor NR2E3 to suppress the development of cone photoreceptors. Brain Res: doi:10.1016/j.brainres.2008.01.028

Stern CD (2005) The chick: a great model system becomes even greater. Dev Cell 8:9-17

Umesono K, Evans RM (1989) Determinants of target gene specificity for steroid/thyroid hormone receptors. Cell 57:1139-1146

Wightman B, Ebert B, Carmean N, Weber K, Clever S (2005) The C. elegans nuclear receptor gene fax-1 and homeobox gene unc-42 coordinate interneuron identity by regulating the expression of glutamate receptor subunits and other neuron-specific genes. Dev Biol 287:74-85

Wolkenberg SE, Zhao ZJ, Kapitskaya A, Webber AL, Petrukhin K, Tang YS, Dean DC, Hartman GD, Lindsley CW (2006) Identification of potent agonists of photoreceptor-specific nuclear receptor $(\mathrm{Nr} 2 \mathrm{e} 3)$ and preparation of a radioligand. Bioorg Med Chem Lett 16:5001-5004

Yu RT, Chiang M-Y, Tanabe T, Kobayashi M, Yasuda K, Evans RM, Umesono K (2000) The orphan nuclear receptor Tlx regulates Pax2 and is essential for vision. Proc Natl Acad Sci USA 97:2621-2625 